\section{From Nonlinear to Hamiltonian via Feedback}

\author{
Paulo Tabuada and George J. Pappas
}

\begin{abstract}
Mechanical control systems are an especially interesting and important class of nonlinear control systems. They posses a rich mathematical structure and yet, physical considerations reveal extremely important for the solution of a large class of control problems. In this note, we broaden the applicability of design methodologies developed for mechanical control systems by rendering nonlinear control systems, mechanical by a proper choice of feedback. In particular, we characterize which control systems can be transformed to Hamiltonian control systems by a feedback transformation.
\end{abstract}

Index Terms-Hamiltonian systems, nonlinear systems, feedback transformation.

\section{INTRODUCTION}

Mechanical control systems are a particularly interesting class of nonlinear control systems as they comprise a refined mathematical structure and yet physical insights are extremely useful to guide control design for these systems. Furthermore, many systems are in fact built by mechanical subsystems which also justifies the interest in mechanical control systems from the applications point of view. It is, therefore, without surprise that we witness the growth of a wealth of powerful design methodologies for these systems. Examples include energy shaping methods [1]-[3], specialized controllability notions and tests [4], [5] motion planning and generation [6], among many others. See also the monographs [7], [8] for several design techniques based on the related notion of passivity.

In this note, we broaden the applicability of design tools for mechanical control systems to other classes of systems by proper choices of feedback. More specifically, we will solve the following equivalence problem:

Given a control system, determine if it is possible to transform it to a Hamiltonian control system by a feedback transformation.

We recall that this has been considered one of the open problems in the area of mechanical feedback control systems as described in the following passage from [9]:

"Find other techniques which enable one to use feedback control for mechanical or, indeed, nonmechanical systems, which leave or put the system into Hamiltonian or Lagrangian form."

The structure of this note is as follows. In Section II, we review some elementary notions of symplectic geometry and introduce the notion of Hamiltonian control systems used throughout the note. In Section III, we provide a simple test to determine if a given control system can be rendered Hamiltonian with respect to a given Hamiltonian. In Section IV, we determine sufficient and necessary conditions for the existence of some Hamiltonian and a feedback transformation rendering a control system Hamiltonian. These geometric conditions are then illustrated with an example. Finally, in Section V, we present some topics for further research.

Manuscript received May 19, 2002; revised January 15, 2003. Recommended by Associate Editor J. M. A. Scherpen. This work was supported in part by the National Science Foundation Information Technology Research Grant CCR0121431.

The authors are with the Department of Electrical and Systems Engineering, University of Pennsylvania, Philadelphia, PA 19104 USA (e-mail tabuadap@seas.upenn.edu; pappasg@ seas.upenn.edu).

Digital Object Identifier 10.1109/TAC.2003.815040

\section{HAMILTONIAN CONTROL SYSTEMS}

Several different models of mechanical control systems abound in the literature on control of mechanical control systems. We will adopt what we think to be one of the simplest such models: Hamiltonian control systems. To introduce it, we review some elementary notions of symplectic geometry [10], [11].

A symplectic form $\omega$ on a smooth manifold $M$ is a two-form satisfying the following properties:

1) Nondegeneracy: $\omega(X, Y)=0$ for every $X \in T M$ implies $Y=0$

2) Closedness: $\mathbf{d} \omega=0$

where we have denoted the exterior derivative by $\mathbf{d}$. We also use the notation $\mathbf{i}_{X} \omega$ to represent the contraction of the two-form $\omega$ with the vector field $X$, that is $\left(\mathbf{i}_{X} \omega\right)(Y)=\omega(X, Y)$ for any $Y \in T M$. The first property of the form $\omega$ is required to obtain an isomorphism between $T M$ and $T^{*} M$ from $\omega$. This is achieved by the correspondence $X \mapsto \mathbf{i}_{X} \omega \in T^{*} M$ which allows to associate a unique Hamiltonian vector field $X_{H}$ with any smooth map $H: M \rightarrow \mathbb{R}$ through the equality

$$
\mathbf{i}_{X_{H}} \omega=\mathbf{d} H .
$$

Hamiltonian vector fields conserve energy (the Hamiltonian $H$ ) along their trajectories $c(t)$, that is

$$
\frac{d}{d t} H(c(t))=\left(\mathcal{L}_{X_{H}} H\right) \circ c(t)=0 .
$$

The nondegeneracy condition on $\omega$ also implies that the dimension on $M$ has to be an even number; see, for example, [10]. The closedness condition is required to ensure that the flow $\phi_{t}$ of a Hamiltonian vector field $X_{H}$ respects the symplectic form, that is $\phi_{t}{ }^{*} \omega=\omega$. We now introduce the class of Hamiltonian control systems we will use in this note.

Definition 2.1: Let $M$ be a smooth manifold equipped with symplectic form $\omega$ and let $U$ be the input manifold. A control affine system $F: M \times U \rightarrow T M$

$$
F=X+\sum_{i=1}^{p} Y_{i} u_{i}
$$

is said to be a Hamiltonian control system with Hamiltonian $H$ if the vector field $X$ is Hamiltonian with Hamiltonian $H$.

Within the context of Hamiltonian control systems one could also consider other models, for example, one could consider that the vector fields $Y_{i}$ are also Hamiltonian [12] or even the more general class of port-controlled Hamiltonian systems [13]. However, we will focus on this simple model as the techniques to be developed extend to the other cases.

\section{Can We Achieve a Given Hamiltonian?}

We start by determining if there exists a feedback transformation, that is, a map ${ }^{1} \alpha(x)+\beta(x) v: M \times U \rightarrow U$ with $\beta(x)$ invertible such that the feedback transformed system

$$
X(x)+\sum_{i} Y_{i}(x) \alpha_{i}(x)+\sum_{i, j} Y_{i}(x) \beta_{i j}(x) v_{j}
$$

is Hamiltonian with Hamiltonian $H$. From expression (III.1) it is clear that one only needs to design $\alpha(x)$ to change $X$, so we will simply consider that $\beta(x)$ is the identity map on $U$. This question has the following simple answer.

\footnotetext{
${ }^{1}$ In the current setting where control systems are assumed to be affine in the inputs it is natural to restrict feedback transformations to affine transformations as this ensures that the transformed system is still input affine.
} 
Proposition 3.1: Let $F$ be an affine control system on a smooth manifold $M$ equipped with symplectic from $\omega$ and $H: M \rightarrow \mathbb{R}$ a smooth map. There exists a local feedback transformation rendering $F$ Hamiltonian with Hamiltonian $H$ iff

$$
\mathbf{d} H-\mathbf{i}_{X} \omega \in \operatorname{span}\left\{\mathbf{i}_{Y_{1}} \omega, \mathbf{i}_{Y_{2}} \omega, \ldots, \mathbf{i}_{Y_{p}} \omega\right\}
$$

or, equivalently

$$
\left(\mathbf{d} H-\mathbf{i}_{X} \omega\right) \wedge \mathbf{i}_{Y_{1}} \omega \wedge \mathbf{i}_{Y_{2}} \omega \wedge \cdots \wedge \mathbf{i}_{Y_{p}} \omega=0 .
$$

Proof: Assume that a such a feedback exists, then the feedback transformed system satisfies

$$
\mathbf{i}_{X+\sum_{i=1}^{p} \alpha_{i}(x) Y_{i}(x)} \omega=\mathbf{d} H
$$

which we rewrite as

$$
\mathbf{d} H-\mathbf{i}_{X} \omega=\sum_{i=1}^{p} \alpha_{i} \mathbf{i}_{Y_{i}} \omega
$$

clearly showing that (III.2) is satisfied. Conversely, assume that (III.2) holds, then there are locally defined smooth functions $a_{i}: M \rightarrow \mathbb{R}$ such that

$$
\mathbf{d} H-\mathbf{i}_{X} \omega=\sum_{i=1}^{p} a_{i} \mathbf{i}_{Y_{i}} \omega .
$$

We now define $\alpha$ by the equalities $\alpha_{i}=a_{i}$ which define the desired local feedback.

While the conditions in Proposition 3.1 provide a quick test to determine if one can transform a control system to a Hamiltonian one with a specified $H$, they are not useful if one wants to search for a feedback transformation and also a Hamiltonian. We devote the next section to this problem.

\section{CAN WE ACHIEVE SOME Hamiltonian?}

To provide a solution for the general case where no Hamiltonian is $a$ priori specified we will reshape condition (III.2). We start by making the following additional assumption:

1) The distribution spanned by the input vector fields $Y_{1}, Y_{2}, \ldots, Y_{p}$, denoted by $\Delta$, is locally of constant rank.

\section{A. Geometric Solution}

To develop a geometric solution we introduce the symplectic orthogonal of $\Delta$, which we denote by $\Delta^{\omega}$ and define by

$$
\Delta^{\omega}=\{Z \in T M: \omega(Z, Y)=0 \quad \forall Y \in \Delta\} .
$$

Note that $\Delta^{\omega}$ is locally of constant rank in virtue of assumption 1 and smoothness of $\omega$. By making use of $\Delta^{\omega}$ we can reformulate condition (III.2) to a more useful form. If condition (III.2) is satisfied, then $\mathbf{d} H-$ $\mathbf{i}_{X} \omega=\sum_{i=1}^{p} a_{i} \mathbf{i}_{Y_{i}} \omega$ and contracting this expression with any vector field in $\Delta^{\omega}$ we have

$$
\mathbf{d} H(Z)=\omega(X, Z) \quad \forall Z \in \Delta^{\omega} .
$$

Conversely, if (IV.1) is satisfied, then $\mathbf{d} H=\mathbf{i}_{X} \omega+\gamma$ for some $\gamma \in \operatorname{span}\left\{\mathbf{i}_{Y_{1}} \omega, \mathbf{i}_{Y_{2}} \omega, \ldots, \mathbf{i}_{Y_{p}} \omega\right\}$ which implies condition (III.2) and shows how (III.2) can be equivalently expressed as (IV.1). This expression can also be regarded as a partial differential equation (PDE) whose solution provides the desired Hamiltonian $H$. We now interpret this PDE geometrically by defining the new manifold

$$
\bar{M}=M \times \mathbb{R}
$$

with local coordinates $(x, y)$, where $x$ are coordinates for $M$ and $y$ coordinates for $\mathbb{R}$. We also define the map

$$
\bar{H}=H-y
$$

as well as the vector fields

$$
\bar{Z}=Z \frac{\partial}{\partial x}+\omega(X, Z) \frac{\partial}{\partial y}
$$

defining the distribution $\overline{\Delta^{\omega}}$ on $\bar{M}$. These new objects allow to rewrite (IV.1) as

$$
\mathcal{L}_{\bar{Z}} \bar{H}=0 \quad \forall \bar{Z} \in \overline{\Delta^{\omega}}
$$

which we interpret as the requirement that vector fields $\bar{Z}$ are tangent to the submanifold $(\bar{H})^{-1}(0)$ of $\bar{M}$. Thus, we see that, in this geometric interpretation of PDE (IV.1), finding a Hamiltonian $H$ is equivalent to finding a submanifold of $\bar{M}$, implicitly defined by a map $\bar{H}: \bar{M} \rightarrow \mathbb{R}$, such that $\bar{\Delta}^{\omega}$ is contained in the tangent space of $(\bar{H})^{-1}(0)$. Furthermore, $\bar{H}$ must also satisfy $(\partial \bar{H} / \partial y) \neq 0$ which ensures, via the implicit function theorem, that $\bar{H}$ defines a function on $M$, the desired Hamiltonian $H$. Necessary and sufficient conditions for the local existence of such a map $\bar{H}$ are given in the next result which can be interpreted as providing geometric conditions for the integrability of PDE (IV.1):

Theorem 4.1: Let $F$ be an affine control system on a smooth manifold $M$ with symplectic form $\omega$ and denote by $\mathcal{C}$ and $\overline{\mathcal{C}}$ the involutive closures of $\Delta^{\omega}$ and $\overline{\Delta^{\omega}}$ which we assume to be regular. There exists a locally defined map $H: M \rightarrow \mathbb{R}$ and a local feedback transformation rendering $F$ Hamiltonian with Hamiltonian $H$ iff

$$
\operatorname{dim}(\mathcal{C})=\operatorname{dim}(\overline{\mathcal{C}}) .
$$

Proof: In view of the discussion preceding Theorem 4.1, it suffices to show that (IV.2) is sufficient and necessary for the existence of the map $\bar{H}: \bar{M} \rightarrow \mathbb{R}$ satisfying $(\partial \bar{H} / \partial y) \neq 0$ and $\overline{\Delta^{\omega}} \subseteq T\left((\bar{H})^{-1}(0)\right)$. To show necessity assume the existence of $\bar{H}=\bar{H}-y$ satisfying $\overline{\Delta^{\omega}} \subseteq T\left((\bar{H})^{-1}(0)\right)$. Let $i: M \rightarrow \bar{M}$ be the map $i(x)=(x, H(x))$ and note that

$$
\begin{aligned}
T_{x} i \cdot Z(x) & =Z(x) \frac{\partial}{\partial x}+T_{x} H \cdot Z(x) \frac{\partial}{\partial y} \\
& =Z(x) \frac{\partial}{\partial x}+\omega(X, Z) \frac{\partial}{\partial y} \\
& =\bar{Z}(x, H(x)) \\
& =\bar{Z} \circ i(x)
\end{aligned}
$$

where the second equality follows from (IV.1). This shows that the vector fields $Z$ are $i$-related to the vector fields $\bar{Z}$. Since if $Z_{i}$ is $i$-related to $\bar{Z}_{i}$ and $Z_{j}$ is $i$-related to $\bar{Z}_{j}$, the bracket $\left[Z_{i}, Z_{j}\right]$ is $i$-related to $\left[\bar{Z}_{i}, \bar{Z}_{j}\right]$ (see [14]), it follows by induction that $\operatorname{dim}(\mathcal{C})=\operatorname{dim}(\overline{\mathcal{C}})$.

Sufficiency is proved by applying Frobenius theorem to $\overline{\mathcal{C}}$ (which is regular by assumption) to ensure the existence of a submanifold $N$ of $\bar{M}$ to which the vector fields in $\overline{\Delta^{\omega}}$ are tangent. Furthermore, Frobenius theorem also ensures that this submanifold is locally described by the zero level of a smooth map $\bar{H}: M \rightarrow \mathbb{R}$ [14]. It remains to show that $(\partial \bar{H} / \partial y) \neq 0$. We proceed by contradiction assuming that $\operatorname{dim}(\mathcal{C})=\operatorname{dim}(\overline{\mathcal{C}})$ and $(\partial \bar{H} / \partial y)=0$. Then the vector field $\bar{Z}=0(\partial / \partial x)+1(\partial / \partial y) \in \operatorname{ker}(\mathbf{d} \bar{H})=\operatorname{ker}((\partial \bar{H} / \partial x) \mathbf{d} x+$ $(\partial \bar{H} / \partial y) \mathbf{d} y)$. This shows that $\operatorname{dim}(\overline{\mathcal{C}})$ is at least greater them $\operatorname{dim}(\mathcal{C})$ by one, a contradiction.

Theorem 4.1 gives necessary and sufficient conditions for the existence of a solution to PDE (IV.1). However, solving PDEs is, in general, a hard problem but a necessary one as they appear in several control design problems for mechanical control systems [2], [3].

As an immediate consequence of Theorem 4.1 we see that in the case $\operatorname{dim}\left(\Delta^{\omega}\right)=1$, that is, control system $F$ has $2 m-1$ inputs and $\operatorname{dim}(M)=2 m$, condition (IV.2) is automatically satisfied.

Corollary 4.2: Let $F$ be an affine control system on a smooth manifold $M$ of dimension $2 m$ with symplectic form $\omega$ and $2 m-1$ inputs. 
Then, there exists a locally defined map $H: M \rightarrow \mathbb{R}$ and a local feedback transformation rendering $F$ Hamiltonian with Hamiltonian $H$.

The discussion so far has assumed that the symplectic form $\omega$ has been a priori specified. However, this is not necessary in virtue of Darboux theorem [10] which asserts that, locally, every symplectic manifold of dimension $2 \mathrm{~m}$ is symplectomorphic (diffeomorphic by a diffeomorphism that preserves the symplectic forms) to $\mathbb{R}^{2 m}$ with symplectic form

$$
\omega=\sum_{i=1}^{m} \mathbf{d} x_{i} \wedge \mathbf{d} y_{i}
$$

expressed in coordinates $\left(x_{1}, x_{2}, \ldots, x_{m}, y_{1}, y_{2}, \ldots, y_{m}\right)$ for $\mathbb{R}^{2 m}$. Thus, we see that if a control system can be locally rendered Hamiltonian with respect to the form $\omega$, then by a change of coordinates, it is also Hamiltonian with respect to any other symplectic form.

\section{B. An Alternative Characterization}

The conditions for the existence of a Hamiltonian and a feedback transformation given in Theorem 4.1 require the computation of several objects such as $\Delta^{\omega}, \overline{\Delta^{\omega}}, \mathcal{C}, \overline{\mathcal{C}}$, etc. However, some of these objects contain some degree of redundancy and we will now see how one can verify the conditions of Theorem 4.1 in a more efficient way. In particular, we shall take advantage of the special form of the vector fields in $\overline{\Delta^{\omega}}$. From the expression of $\left[\overline{Z_{i}}, \overline{Z_{j}}\right]$ in local coordinates

$$
\begin{aligned}
{\left[\overline{Z_{i}}, \overline{Z_{j}}\right]=\left[\begin{array}{cc}
\frac{\partial Z_{j}}{\partial x} & 0 \\
\mathbf{d} \omega\left(X, Z_{j}\right) & 0
\end{array}\right]\left[\begin{array}{c}
Z_{i} \\
\omega\left(X, Z_{i}\right)
\end{array}\right] } & \\
& -\left[\begin{array}{cc}
\frac{\partial Z_{i}}{\partial x} & 0 \\
\mathbf{d} \omega\left(X, Z_{i}\right) & 0
\end{array}\right]\left[\begin{array}{c}
Z_{j} \\
\omega\left(X, Z_{j}\right)
\end{array}\right]
\end{aligned}
$$

we see that $\left[\overline{Z_{i}}, \overline{Z_{j}}\right]$ is given by

$$
\left[Z_{i}, Z_{j}\right] \frac{\partial}{\partial x}+\left(\mathcal{L}_{Z_{i}} \omega\left(X, Z_{j}\right)-\mathcal{L}_{Z_{j}} \omega\left(X, Z_{i}\right)\right) \frac{\partial}{\partial y} .
$$

We now rewrite $\mathcal{L}_{Z_{i}} \omega\left(X, Z_{j}\right)-\mathcal{L}_{Z_{j}} \omega\left(X, Z_{i}\right)$ as

$$
-\mathbf{d}\left(\mathbf{i}_{Z_{j}} \mathbf{i}_{X} \omega\right)\left(Z_{i}\right)-\mathcal{L}_{Z_{j}} \omega\left(X, Z_{i}\right)
$$

which by the Cartan magic formula [14] becomes

$$
\begin{aligned}
& -\left(\mathcal{L}_{Z_{j}} \mathbf{i}_{X} \omega-\mathbf{i}_{Z_{j}} \mathbf{d i} \mathbf{i}_{X} \omega\right)\left(Z_{i}\right)-\mathcal{L}_{Z_{j}} \omega\left(X, Z_{i}\right) \\
= & -\mathbf{i}_{Z_{i}} \mathcal{L}_{Z_{j}} \mathbf{i}_{X} \omega+\mathbf{i}_{Z_{i}} \mathbf{i}_{Z_{j}} \mathbf{d} \mathbf{i}_{X} \omega-\mathcal{L}_{Z_{j}} \omega\left(X, Z_{i}\right) \\
= & \mathcal{L}_{Z_{j}} \mathbf{i}_{Z_{i}} \mathbf{i}_{X} \omega-\mathbf{i}_{Z_{i}} \mathcal{L}_{Z_{j}} \mathbf{i}_{X} \omega+\mathbf{i}_{Z_{i}} \mathbf{i}_{Z_{j}} \mathbf{d} \mathbf{i}_{X} \omega .
\end{aligned}
$$

Using the fact that $\mathcal{L}_{Z_{j}} \mathbf{i}_{Z_{i}} \gamma-\mathbf{i}_{Z_{i}} \mathcal{L}_{Z_{j}} \gamma=\gamma\left(\left[Z_{i}, Z_{j}\right]\right)$ for any one-form $\gamma$ we obtain

$$
\omega\left(X,\left[Z_{i}, Z_{j}\right]\right)+\mathbf{i}_{Z_{i}} \mathbf{i}_{Z_{j}} \mathbf{d i} \mathbf{i}_{X} \omega .
$$

Expression (IV.5) allows to formulate the following alternative version of Theorem 4.1.

Theorem 4.3 (Alternative Characterization): Let $F$ be an affine control system on a smooth manifold $M$ with symplectic form $\omega$. There exists a locally defined map $H: M \rightarrow \mathbb{R}$ and a local feedback transformation rendering $F$ Hamiltonian with Hamiltonian $H$ iff

$$
\mathbf{i}_{Z_{i}} \mathbf{i}_{Z_{j}} \mathbf{d} \mathbf{i}_{X} \omega=0 \quad \forall Z_{i}, Z_{j} \in \Delta^{\omega} .
$$

Proof: As we have seen in the proof of Theorem 4.1, existence of $H$ and the feedback transformation implies that every vector field $Z \in \Delta^{\omega}$ is $i$-related to the vector field $\bar{Z} \in \overline{\Delta^{\omega}}$ for $i(x)=(x, H(x))$. This, in turn, implies that $\left[Z_{i}, Z_{j}\right]$ is also $i$-related to $\left[\overline{Z_{i}}, \overline{Z_{j}}\right]$ leading to

$$
\left[\overline{Z_{i}}, \overline{Z_{j}}\right]=\left[Z_{i}, Z_{j}\right] \frac{\partial}{\partial x}+\omega\left(X,\left[Z_{i}, Z_{j}\right]\right) \frac{\partial}{\partial y} .
$$

Comparing this expression with (IV.5), we see that we necessarily have $\mathbf{i}_{Z_{i}} \mathbf{i}_{Z_{j}} \mathbf{d} \mathbf{i}_{X} \omega=0$.

To show sufficiency we note that if $\left[Z_{i}, Z_{j}\right]=\sum_{l} a_{l} Z_{j}$ for $Z_{i}, Z_{j}, Z_{l} \in \mathcal{C}$ and smooth real valued functions $a_{l}$, it follows by (IV.5) that $\left[\overline{Z_{i}}, \overline{Z_{j}}\right]$ equals

$$
\begin{aligned}
& {\left[Z_{i}, Z_{j}\right] \frac{\partial}{\partial x}+\left(\omega\left(X,\left[Z_{i}, Z_{j}\right]\right)+\mathbf{i}_{Z_{i}} \mathbf{i}_{Z_{j}} \mathbf{d i}_{X} \omega\right) \frac{\partial}{\partial y} } \\
= & \sum_{l} a_{l} Z_{l} \frac{\partial}{\partial x}+\left(\omega\left(X, \sum_{l} a_{l} Z_{l}\right)+\mathbf{i}_{Z_{i}} \mathbf{i}_{Z_{j}} \mathbf{d i} \mathbf{i}_{X} \omega\right) \frac{\partial}{\partial y} \\
= & \sum_{l} a_{l} Z_{l} \frac{\partial}{\partial x}+\left(\sum_{l} a_{l} \omega\left(X, Z_{l}\right)+\mathbf{i}_{Z_{i}} \mathbf{i}_{Z_{j}} \mathbf{d i} \mathbf{i}_{X} \omega\right) \frac{\partial}{\partial y} \\
= & \sum_{l} a_{l} \overline{Z_{l}}+\mathbf{i}_{Z_{i}} \mathbf{i}_{Z_{j}} \mathbf{d i}_{X} \omega \frac{\partial}{\partial y} .
\end{aligned}
$$

The assumption $\mathbf{i}_{Z_{i}} \mathbf{i}_{Z_{j}} \mathbf{d i} \mathbf{i}_{X} \omega=0$ now allows to conclude that $\left[Z_{i}, Z_{l}\right]=\sum a_{l} Z_{l} \Rightarrow\left[\overline{Z_{i}}, \overline{Z_{l}}\right]=\sum a_{l} \overline{Z_{l}}$ and an induction argument shows that $\operatorname{dim}(\mathcal{C})=\operatorname{dim}(\overline{\mathcal{C}})$ which by Theorem 4.1 implies the existence of $H$ and the desired feedback transformation.

This formulation also allows to see that Corollary 4.2 is a simple consequence of the skew-symmetry of $\omega$. If $\operatorname{dim}\left(\Delta^{\omega}\right)=1$ we have that for any $Z_{i}, Z_{j} \in \Delta^{\omega}, Z_{i}=\lambda Z_{j}$ for a smooth real valued map $\lambda$ and $\left(\mathbf{d i}_{X} \omega\right)\left(Z_{i}, \lambda Z_{i}\right)=\lambda\left(\mathbf{d i}_{X} \omega\right)\left(Z_{i}, Z_{i}\right)=0$.

The necessary steps to determine the existence of a solution to PDE (IV.1) can now be resumed to the following.

1) Compute a basis for the symplectic orthogonal $\Delta^{\omega}$ of $\Delta$

$$
\Delta^{\omega}=\{Z \in T M: \omega(Z, Y)=0 \quad \forall Y \in \Delta\} .
$$

2) Check if $\mathbf{i}_{Z_{i}} \mathbf{i}_{Z_{j}} \mathbf{d i} \mathbf{i}_{X} \omega=0$ for every $Z_{i}, Z_{j}$ in the basis of $\Delta^{\omega}$ computed in step 1).

If Theorem 4.1 is satisfied, then a solution to (IV.1) must be obtained in order to determine the feedback transformation. If $H$ is such a solution, we determine the feedback transformation by computing the smooth functions $a_{i}$ satisfying

$$
\mathbf{d} H-\mathbf{i}_{X} \omega=\sum_{i=1}^{p} a_{i} \mathbf{i}_{Y_{i}} \omega .
$$

These functions allow to determine the term $\alpha(x)$ of the feedback transformation $\alpha(x)+\beta(x) v$ by the equalities $\alpha_{i}=a_{i}$. The term $\beta(x)$ can be taken as the identity on $U$ or any other invertible (pointwise) linear map from $U$ to $U$.

\section{Example}

We now provide an example of the previously introduced methodology. Consider the following control system:

$$
\begin{aligned}
\dot{x}_{1} & =x_{2} x_{3}+x_{2} u_{1} \\
\dot{x}_{2} & =x_{1} x_{2}^{2}+\frac{1}{2} x_{3}^{2} \\
\dot{x}_{3} & =x_{1} x_{2} x_{4}+x_{2} x_{3} u_{2} \\
\dot{x}_{4} & =x_{1} x_{3}
\end{aligned}
$$

on $\mathbb{R}^{4}$ with symplectic form $\omega=\mathbf{d} x_{1} \wedge \mathbf{d} x_{2}+\mathbf{d} x_{3} \wedge \mathbf{d} x_{4}$. In this case, we have

$$
X=\left[\begin{array}{c}
x_{2} x_{3} \\
x_{1} x_{2}^{2}+\frac{1}{2} x_{3}^{2} \\
x_{1} x_{2} x_{4} \\
x_{1} x_{3}
\end{array}\right] \quad Y_{1}=\left[\begin{array}{c}
x_{2} \\
0 \\
0 \\
0
\end{array}\right] \quad Y_{2}=\left[\begin{array}{c}
0 \\
0 \\
x_{2} x_{3} \\
0
\end{array}\right]
$$

and $\Delta=\operatorname{span}\left\{Y_{1}, Y_{2}\right\}$. We now follow the steps outlined in the previous section.

1) The symplectic orthogonal of $\Delta$ is obtained by first computing

$$
\begin{aligned}
& \mathbf{i}_{Y_{1}} \omega=x_{2} \mathbf{d} x_{2} \\
& \mathbf{i}_{Y_{2}} \omega=x_{2} x_{3} \mathbf{d} x_{4}
\end{aligned}
$$


and then determining $\Delta^{\omega}$ as the annihilating distribution of $\operatorname{span}\left\{\mathbf{i}_{Y_{1}} \omega, \mathbf{i}_{Y_{2}} \omega\right\}$. Distribution $\Delta^{\omega}$ is then given by the span of

$$
Z_{1}=\left[\begin{array}{l}
1 \\
0 \\
0 \\
0
\end{array}\right] \quad Z_{2}=\left[\begin{array}{l}
0 \\
0 \\
1 \\
0
\end{array}\right]
$$

2) We now compute

$$
\begin{aligned}
\mathbf{i}_{X} \omega=-\left(\frac{1}{2} x_{3}^{2}+x_{1} x_{2}^{2}\right) \mathbf{d} x_{1}+x_{2} x_{3} \mathbf{d} & x_{2} \\
& -x_{1} x_{3} \mathbf{d} x_{3}+x_{1} x_{2} x_{4} \mathbf{d} x_{4}
\end{aligned}
$$

which by differentiation gives

$$
\begin{aligned}
\mathbf{d i}_{X} \omega= & -2 x_{1} x_{2} \mathbf{d} x_{2} \wedge \mathbf{d} x_{1}-x_{3} \mathbf{d} x_{3} \wedge \mathbf{d} x_{1} \\
& +x_{2} \mathbf{d} x_{3} \wedge \mathbf{d} x_{2}-x_{3} \mathbf{d} x_{1} \wedge \mathbf{d} x_{3} \\
& +x_{2} x_{4} \mathbf{d} x_{1} \wedge \mathbf{d} x_{4}+x_{1} x_{4} \mathbf{d} x_{2} \wedge \mathbf{d} x_{4} \\
= & -2 x_{1} x_{2} \mathbf{d} x_{2} \wedge \mathbf{d} x_{1}+x_{2} \mathbf{d} x_{3} \wedge \mathbf{d} x_{2} \\
& +x_{2} x_{4} \mathbf{d} x_{1} \wedge \mathbf{d} x_{4}+x_{1} x_{4} \mathbf{d} x_{2} \wedge \mathbf{d} x_{4}
\end{aligned}
$$

and evaluating $\operatorname{di}_{X} \omega$ on $Z_{1}$ and $Z_{2}$ :

$$
\begin{aligned}
\mathbf{i}_{Z_{2}} \mathbf{d} \mathbf{i}_{X} \omega & =x_{2} \mathbf{d} x_{2} \\
\mathbf{i}_{Z_{1}} \mathbf{i}_{Z_{2}} \mathbf{d} \mathbf{i}_{X} \omega & =0
\end{aligned}
$$

shows, via Theorem 4.3, that a Hamiltonian and a feedback transformation exist.

To obtain $H$ one has to solve (IV.1), which in this case results in

$$
\begin{aligned}
& \frac{\partial H}{\partial x_{1}}=-\frac{1}{2} x_{3}^{2}-x_{1} x_{2}^{2} \\
& \frac{\partial H}{\partial x_{3}}=-x_{1} x_{3} .
\end{aligned}
$$

It suffices to solve the first equation to obtain

$$
H=-\frac{1}{2}\left(x_{1}^{2} x_{2}^{2}+x_{1} x_{3}^{2}\right) .
$$

One now computes $\mathbf{d} H-\mathbf{i}_{X} \omega=\left(x_{1}^{2} x_{2}-x_{2} x_{3}\right) \mathbf{d} x_{2}-x_{1} x_{2} x_{4} \mathbf{d} x_{4}$ which can be written as $\mathbf{d} H-\mathbf{i}_{X} \omega=a_{1} \mathbf{i}_{Y_{1}} \omega+a_{2} \mathbf{i}_{Y_{2}} \omega$ for

$$
a_{1}=x_{1}^{2}-x_{3} \quad a_{2}=\frac{1}{x_{3}} x_{1} x_{4} .
$$

These functions now allow to define the feedback transformation as $\alpha_{i}(x)=a_{i}(x)$ and for $\beta(x)$ we simply use the identity on $U$. The feedback transformed system is now of the form

$$
\begin{aligned}
\dot{x}_{1} & =\frac{\partial H}{\partial x_{2}}+x_{2} u_{1} \\
\dot{x}_{2} & =-\frac{\partial H}{\partial x_{1}} \\
\dot{x}_{3} & =\frac{\partial H}{\partial x_{4}}+x_{2} x_{3} u_{2} \\
\dot{x}_{4} & =-\frac{\partial H}{\partial x_{3}}
\end{aligned}
$$

revealing its Hamiltonian structure.

\section{CONCLUSION}

In this note, we addressed the problem of rendering a nonlinear control system Hamiltonian by a proper choice of feedback. We showed that the solution is given by the solution of a PDE and provided sufficient and necessary conditions for the local existence of solutions.
These results enlarge the class of systems to which powerful control design methods developed for mechanical systems are applicable.

Many related problems remain open. When we cannot perform such a feedback transformation it may still be possible to extract a quotient (an abstraction, see, for example, [15] and [16]) or a subsystem that is mechanical, or that can be rendered mechanical by feedback. This would allow to synthesize controllers for part of the variables by making use of techniques developed for mechanical control systems.

\section{REFERENCES}

[1] A. M. Bloch and J. E. M. N. E. Leonard, "Controlled lagrangians and the stabilization of mechanical systems I: The first matching theorem," IEEE Trans. Automat. Contr., vol. 45, pp. 2253-2270, Dec. 2000.

[2] A. M. Bloch, D. Chang, and J. E. M. N. E. Leonard, "Controlled Lagrangians and the stabilization of mechanical systems II: Potential shaping," IEEE Trans. Automat. Contr., vol. 46, pp. 1556-1571, Oct. 2001 .

[3] R. Ortega, A. van der Schaft, B. Maschke, and G. Escobar, "Interconnection and damping assignment passivity-based control of port-controlled Hamiltonian systems," Automatica, vol. 38, no. 4, pp. 585-596, Apr. 2002.

[4] A. Lewis and R. M. Murray, "Configuration controllability of simple mechanical control systems," SIAM J. Control Optim., vol. 35, no. 3, pp. 766-790, May 1997.

[5] F. Bullo and K. M. Lynch, "Kinematic controllability for decoupled trajectory planning in underactuated mechanical systems," IEEE Trans. Robot. Automa., vol. 17, pp. 402-412, Aug. 2001.

[6] A. D. L. F. Bullo and N. E. Leonard, "Controllability and motion algorithms for underactuated Lagrangian systems on lie groups," IEEE Trans. Automat. Contr., vol. 45, pp. 1437-1454, Aug. 2000.

[7] R. Ortega and P. J. Nicklasson, Passivity-Based Control of Euler-Lagrange Systems, ser. Communications and Control Engineering. New York: Springer-Verlag, Oct. 1998.

[8] R. Sepulchre, M. Jankovic, and P. V. Kokotovic, Constructive Nonlinear Control, ser. Communications and Control Engineering. New York: Springer-Verlag, Jan. 1997.

[9] A. M. Bloch, N. E. Leonard, and J. E. Marsden, "Mechanical feedback control systems," in Open Problems in Mathematical Systems and Control Theory, V. D. Blondel, E. D. Sontag, M. Vidyasagar, and J. C. Willems, Eds. New York: Springer-Verlag, 1999, pp. 39-44.

[10] A. C. da Silva, Lectures on Symplectic Geometry, ser. Lecture Notes in Mathematics. New York: Springer-Verlag, 2001, vol. 1764.

[11] J. E. Marsden and T. S. Ratiu, Introduction to Mechanics and Symmetry, ser. Texts in Applied Mathematics. New York: Springer-Verlag, 1999.

[12] A. J. van der Schaft, "Hamiltonian dynamics with external forces and observations," Math. Syst. Theory, vol. 15, pp. 145-168, 1982.

[13] A. van der Schaft, $L_{2}$-Gain and Passivity Techniques in Nonlinear Control, ser. Lecture Notes in Control and Information Sciences. London, U.K.: Springer-Verlag, 1996, vol. 218.

[14] R. Abraham, J. Marsden, and T. Ratiu, Manifolds, Tensor Analysis and Applications, ser. Applied Mathematical Sciences. New York: Springer-Verlag, 1988.

[15] G. J. Pappas and S. Simic, "Consistent hierarchies of affine nonlinear systems," IEEE Trans. Automat. Contr., vol. 47, pp. 745-756, May 2002.

[16] P. Tabuada and G. J. Pappas. Abstractions of Hamiltonian control systems. Automatica [Online]. Available: http://www.seas.upenn.edu/ rabuadap 